\title{
Observation of Landau levels of Dirac fermions in graphite
}

\author{
GUOHONG LI AND EVA Y. ANDRE|* \\ Department of Physics and Astronomy, Rutgers University, Piscataway, New Jersey 08854, USA \\ *e-mail: eandrei@physics.rutgers.edu
}

The unique electronic behaviour of monolayer and bilayer graphene $e^{1,2}$ is a result of the unusual quantum-relativistic characteristics of the so-called 'Dirac fermions' (DFs) that carry charge in these materials. Although DFs in monolayer graphene move as if they were massless, and in bilayer graphene they do so with non-zero mass, all DFs show chirality, which gives rise to an unusual Landau level (LL) energy spectrum ${ }^{3-11}$ and the observation of an anomalous quantum Hall effect in both types of graphene $e^{4,5,8}$. Here we report low-temperature scanning tunnelling spectra of graphite subjected to a magnetic field of up to $12 \mathrm{~T}$, which provide the first direct observations of the LLs that produce such behaviour. Unexpectedly, we find evidence for the coexistence of both massless and massive DFs in graphite, and confirm the quantum-relativistic nature of these quasiparticles through the appearance of a zero-energy $\mathrm{LL}$.

The dynamics of electrons inside a material is usually described within the framework of non-relativistic quantum mechanics. For parabolic energy bands the energy-momentum dispersion $E=E_{ \pm} \pm \hbar^{2} k^{2} / 2 m^{*}$ resembles that of non-relativistic free particles with an effective mass $m^{*}$ arising from interactions with the lattice. Here the energy is measured relative to the bottom of the conduction band, $E_{+}=E_{\mathrm{C}}$, for electron-like $(+)$ particles, or to the top of the valence band, $E_{-}=E_{\mathrm{V}}$, for holelike $(-)$ particles. In the presence of a magnetic field, $B$, the energy for motion perpendicular to the field is quantized in a series of equally spaced LLs:

$$
E_{n}=E_{ \pm} \pm \hbar \omega_{c}(n+1 / 2), \quad n=0,1,2, \ldots
$$

where $\hbar$ is Planck's constant, $\omega_{c}=e B / m^{*}$ the cyclotron frequency and $e$ the electron charge.

This standard model fails radically when applied to electrons in graphene-a two-dimensional layer of carbon atoms tightly packed into a honeycomb structure ${ }^{1,2}$ consisting of two distinct triangular Bravais sublattices. This arrangement has non-trivial consequences: it endows the electronic states with internal degrees of freedom, isospin, leading to well-defined chirality, and produces a highly diminished Fermi 'surface' consisting of two inequivalent 'Dirac points' (DPs), where the valence and conduction bands touch. The hamiltonian describing the low-energy excitation is that of a $(2+1)$ relativistic quantum system ${ }^{12,13}$ described by the Dirac equation for particles with zero mass and spin $1 / 2$. The solutions, consisting of particle-antiparticle pairs with opposite chiralities, resemble relativistic massless DFs in every way except that they move at the Fermi velocity $v_{\mathrm{F}} \sim c / 300$ instead of the speed of light, $c$. Thus, the low-energy excitation spectrum measured with respect to the DP is linear in momentum, $\hbar k$, with $E(k)= \pm v_{\mathrm{F}} \hbar k$.
The relativistic nature of massless DFs is revealed through an unusual LL energy sequence, which consists of a field-independent state at zero energy followed by a sequence of levels with squareroot dependence in both field and level index, instead of the usual linear dependence:

$$
E_{n}=\operatorname{sgn}(n) \sqrt{2 e \hbar v_{\mathrm{F}}^{2}|n| B}, \quad n=\ldots-2,-1,0,1,2, \ldots
$$

Here the energy is measured relative to the DP energy, $E_{\mathrm{D}}$. The unusual appearance of a zero-energy level for $n=0$ is a direct consequence of chirality. This level has the same degeneracy as the $n \neq 0$ levels, but it is the only one independent of field.

When two graphene layers stack to form a bilayer, the interlayer coupling leads to the appearance of band mass but it does not open a gap at the DP. Here the quasiparticles are still chiral and the LL spectrum takes the form ${ }^{8-10} E_{n}= \pm \hbar \omega_{c} \sqrt{n(n-1)}, n=0,1,2, \ldots$. This sequence is linear in field, similar to the standard case, but it contains an additional zero-energy level, which is independent of field. Here, as in the case for massless DFs, the zero-energy level is a consequence of chirality, but its degeneracy is now double that of the $n \neq 0$ levels. To facilitate the data analysis we will use an alternative form:

$$
E_{n}=\operatorname{sgn}(n) \hbar \omega_{\mathrm{c}} \sqrt{|n|(|n|+1)}, \quad n=\ldots-2,-1,0,1,2, \ldots
$$

This form gives the same LL spectrum, including the double degeneracy of the zero-energy level. Indirect evidence of a zeroenergy LL was recently obtained through the appearance of quantum Hall effect anomalies observed in single- and bilayer graphene $e^{4,5,8}$ as well as in highly oriented pyrolytic graphite $(\mathrm{HOPG})^{14,15}$. However, to observe the LL directly, a more specialized technique is required. Scanning tunnelling spectroscopy (STS) enables selection of specific energy levels by varying the voltage bias between sample and tip, and the tunnelling current gives direct access to the density of states. Obtaining tunnelling spectra on graphene films is challenging because the films are quite small, and locating them in the field of view of a low-temperature scanning tunnelling microscope can be daunting. Furthermore, most substrate materials interfere significantly with the intrinsic energy levels ${ }^{16,17}$. Surprisingly, we found that the LLs of both massless and massive DFs can be accessed on the surface of bulk HOPG samples, suggesting decoupling of the surface states from the bulk. This is in contrast to STS results on Kish graphite, where the LL spectra are consistent with bulk ${ }^{18}$.

Figure 1a shows tunnelling spectra measured on the surface of HOPG at $4.4 \mathrm{~K}$ in magnetic fields up to $12 \mathrm{~T}$. Similar spectra on HOPG were recently reported by another group ${ }^{19}$. We note that all 

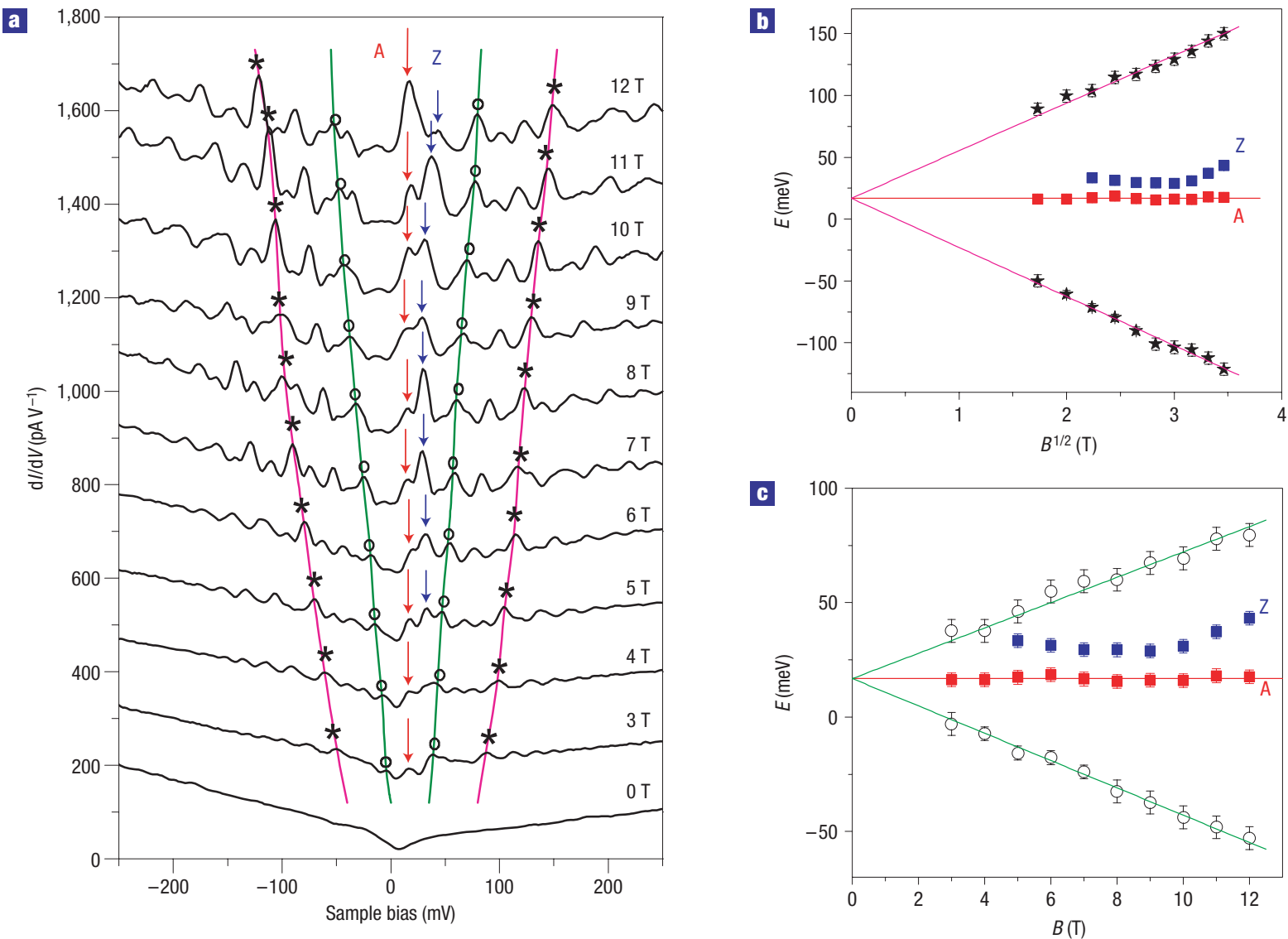

Figure 1 Tunnelling spectra on graphite. a, Magnetic-field dependence of tunnelling spectra on a graphite surface at 4.4 K. All peaks, except the two labelled A and Z, show strong field dependence, shifting away from zero bias with increasing field. The spectra are shifted vertically by $150 \mathrm{pAV}^{-1}$ for clarity. $\mathbf{b}$, Landau levels marked with stars in a show square-root field dependence and extrapolate to the energy of peak $A$ at zero field. Also shown is the field dependence of peaks $A$ and $Z$. c, Landau levels marked with circles in a show linear field dependence and extrapolate to the energy of peak $A$ at zero field. The error bars in $\mathbf{b}$ and $\mathbf{c}$ correspond to the line-widths of the peaks in $\mathbf{a}$.

peaks, except the two labelled $A$ and $Z$, shift away from the origin with increasing field. Peak $A$ is independent of field, whereas peak $Z$ shows weak non-monotonic field dependence consistent with that previously associated with the $n=0,-1$ bulk LLs of graphite ${ }^{18}$. Analysing the field dependence of the spectra in Fig. 1a, we find two families of peaks: one with square-root dependence and the other linear. Two peaks in each family are highlighted with stars and open circles for the square-root and linear sequences respectively. The square-root field dependence of the former becomes evident by plotting the peak positions against $B^{1 / 2}$ in Fig. $1 \mathrm{~b}$. We note that the positive energy sequence has positive slope, as expected of electron-like excitations, whereas the negative slope of the negative energy sequence indicates holelike behaviour. The field dependence of the levels in both sequences extrapolates to the same zero-field value, $16.8 \pm 3 \mathrm{meV}$, implying that, within experimental error, the conduction and valence bands touch and that the intersection point coincides with the field-independent peak A. Comparing with equation (2) suggests that the square-root sequence corresponds to the LLs of massless DFs, in which case we need to include peak A and identify its energy with the DP, $E_{\mathrm{D}}$. A deviation of the DP from zero, also observed in angle-resolved photoemission spectroscopy $(\mathrm{ARPES})^{20}$, is often attributed to substrate charging. The positive value of $E_{\mathrm{D}}$ measured here implies a hole-doped sample.

We now proceed to analyse the dependence on LL index, $n$. From equation (2), the LL energy of massless DFs scales according to $E / B^{1 / 2}$, where $E$ is referred to the DP. We therefore replot in Fig. 2 the spectra against the scaled energy $E / B^{1 / 2}$ with the energy origin shifted to the DP, $E-E_{\mathrm{D}} \rightarrow E$. It is now straightforward to identify a family of peaks that align on the same value of scaled energy. All the aligned peaks are then indexed starting with the $n=0$ level (peak A) at the origin. The unaligned peaks are left out now. When plotting in Fig. $2 c$ the scaled energy of the aligned peaks against $|n|^{1 / 2}$, the square-root dependence on level index becomes evident.

The family of peaks with linear field dependence (Fig. 1c) is considered next. As before, the sequence with positive (negative) energy is attributed to electron-like (holelike) particles. Both sequences extrapolate to the same energy at zero field, indicating that the conduction and valence bands touch. Notably, this energy coincides with the energy $E_{\mathrm{D}}$ found in the extrapolation of the square-root sequence. The linear field dependence implies finite mass ${ }^{21}$, but whether it represents standard particles (equation (1)) or chiral ones (equation (3)) can be determined only by analysing the dependence on LL index. In Fig. 3a we plot the low-field spectra against the scaled variable $E / B$. This scaling leads to the alignment of all peaks associated with massive particles, but those associated with massless particles (square-root sequence) become unaligned. Note that peak $Z$ does not scale with either $B$ or $B^{1 / 2}$, so it is not included in either sequence. To assign an LL index to these peaks, we need to establish whether peak A, which is pinned to the Dirac point, should be considered part of this family. If we 

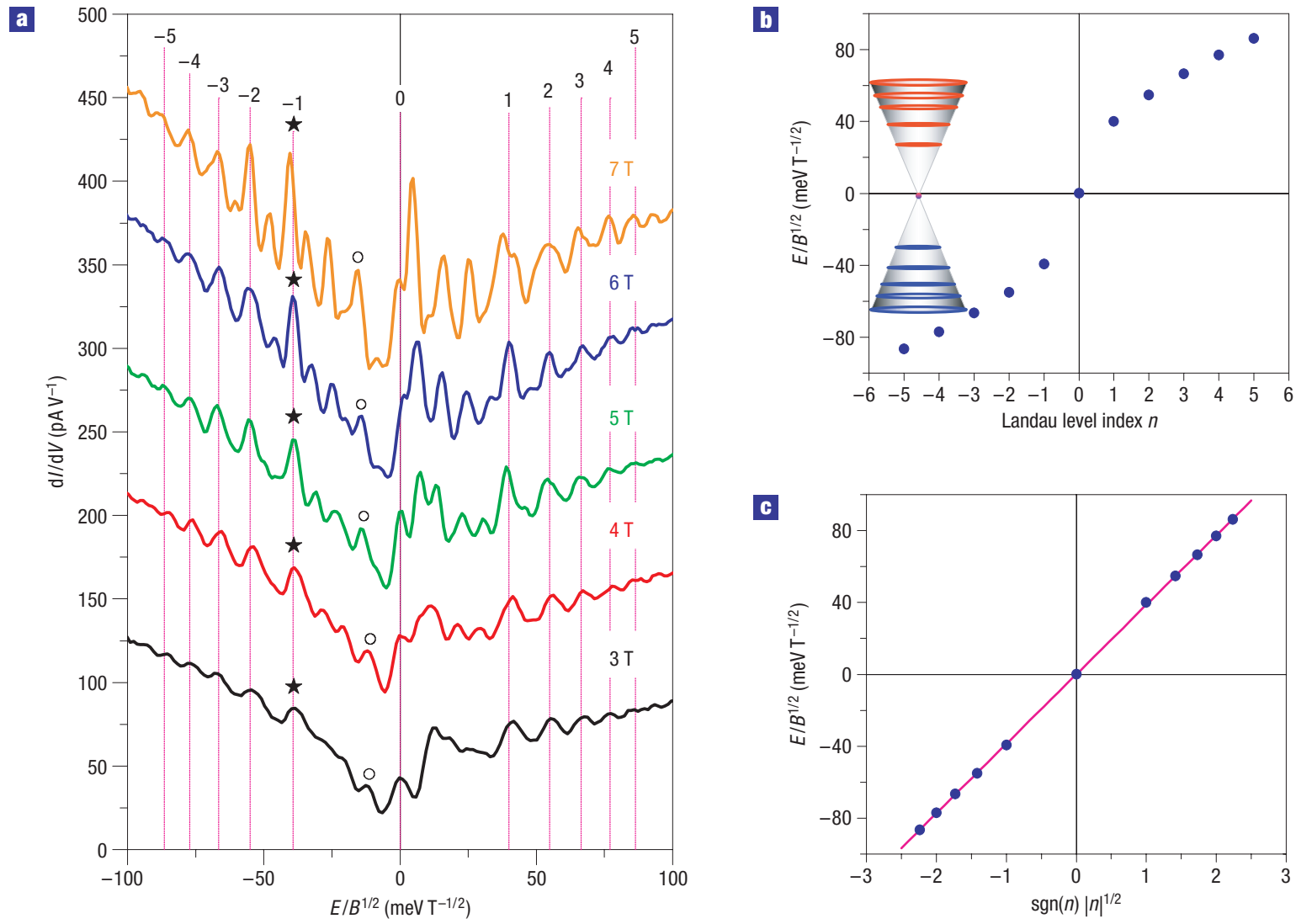

Figure 2 Landau levels of massless Dirac fermions. a, Tunnelling spectra plotted against the reduced energy $E / B^{1 / 2}$. The energy origin is shifted to peak $A$ in Fig. 1 . The peaks marked with stars are aligned after scaling, whereas those marked with circles are not. All aligned peaks are labelled with sequential $L L$ indices. The spectra are shifted vertically by $70 \mathrm{pAV}^{-1}$ for clarity. b. Scaled energy of the aligned peaks plotted against LL index. Inset: Landau-level energy sequence of massless DFs superposed on the Dirac cone. c, Scaled energy levels are linear in $\operatorname{sgn}(n)|n|^{1 / 2}$, as expected for massless DFs. From a comparison of the slope of the linear fit with equation (2) we obtain the Fermi velocity: $v_{\mathrm{F}}=(1.07 \pm 0.05) \times 10^{6} \mathrm{~m} \mathrm{~s}^{-1}$, consistent with that obtained from transport measurements on graphene $e^{4,5}$ and ARPES on graphite ${ }^{20}$.

assume standard massive fermions, then peak A should be left out. In this case the first peaks in the sequence (marked with open circles) have to be labelled $n=-1$ and $n=+1$ for the negative and positive energies, respectively. Any other choice would lead to $E_{\mathrm{V}} \neq E_{\mathrm{C}} \neq E_{\mathrm{D}}$. However, if this assignment is correct then the two $n=0$ levels, marked with arrows in Fig. $3 \mathrm{~b}$, are inexplicably missing. If we assume the spectrum corresponds to massive DFs, then peak A should be included as the $n=0$ level and the other aligned peaks are labelled in ascending order as shown in Fig. 3a. Plotting the scaled energy for this sequence against $(|n|(|n|+1))^{1 / 2}$ in Fig. 3c, we now find agreement with equation (3) in support of the interpretation in terms of massive DFs. From the slope of these data we obtain an effective mass of $m^{*}=(0.028 \pm 0.003) m_{\mathrm{e}}$ for both electrons and holes, where $m_{\mathrm{e}}$ is the free-electron mass. This value is comparable to the hole mass obtained with transport measurements ${ }^{22-24}\left(m^{*}=0.03 m_{\mathrm{e}}\right)$ but is smaller than that obtained with ARPES ${ }^{20}\left(m^{*}=0.069 m_{\mathrm{e}}\right)$.

The data for all resolved LLs, shown in Fig. 4, can be classified into three groups (see also Supplementary Information, Figs S1,S2): massless DFs (Fig. 4a), massive DFs (Fig. 4b) and unidentified peaks (Fig. 4c). A classification of the last group requires detailed band-structure calculations, which will be reported elsewhere. Here we wish only to point out that for the massive electrons and holes in this category the condition $E_{\mathrm{V}}=E_{\mathrm{C}}=E_{\mathrm{D}}$ is generally not valid.

The observation of scanning tunnelling spectra corresponding to massless and massive DFs, together with the fact that their energies extrapolate to the same DP at zero field, is a strong indication of purely two-dimensional quasiparticles. This is expected in samples of few-layer graphene ${ }^{25}$, but is quite surprising for bulk graphite because there, although both types of DF are present ${ }^{20,26}$, the corresponding DPs are shifted with respect to each other by $\sim 50 \mathrm{meV}$. Moreover, the spectral weight of the twodimensional quasiparticles in graphite is negligible compared to that of bulk excitations along the $\mathrm{H}-\mathrm{K}$ direction, and therefore they should not be detectable with a technique, such as STS, which is not momentum selective ${ }^{18}$. The findings reported here strongly suggest that for HOPG samples only a few top layers of the graphite contribute to the observed electronic states.

To understand the origin of the observed two-dimensional behaviour we first consider the tip-induced charging of the graphite surface. The proximity of the tip causes an excess surface charge density ${ }^{27}$ of $n \approx 5 \times 10^{12}\left(V_{\mathrm{b}} / d\right) \mathrm{cm}^{-2}$, where $V_{\mathrm{b}}$ is the bias voltage in volts and $d$ the tip-sample distance in nanometres. For our experiment $(d \sim 8 \mathrm{~nm})$ this causes a shift in Fermi energy $(<1 \mathrm{meV})$ that is too small to affect the analysis. This was confirmed by repeating the experiments for several values of the tip-sample distance (see Supplementary Information, Fig. S3) and finding that the spectra are independent of tip distance for all set currents below $100 \mathrm{pA}$ (the data shown here were taken at $25 \mathrm{pA})$. Only when the currents become much larger (smaller tip-sample distance) do we observe a small shift in Fermi energy together with an increase of up to $10 \%$ 

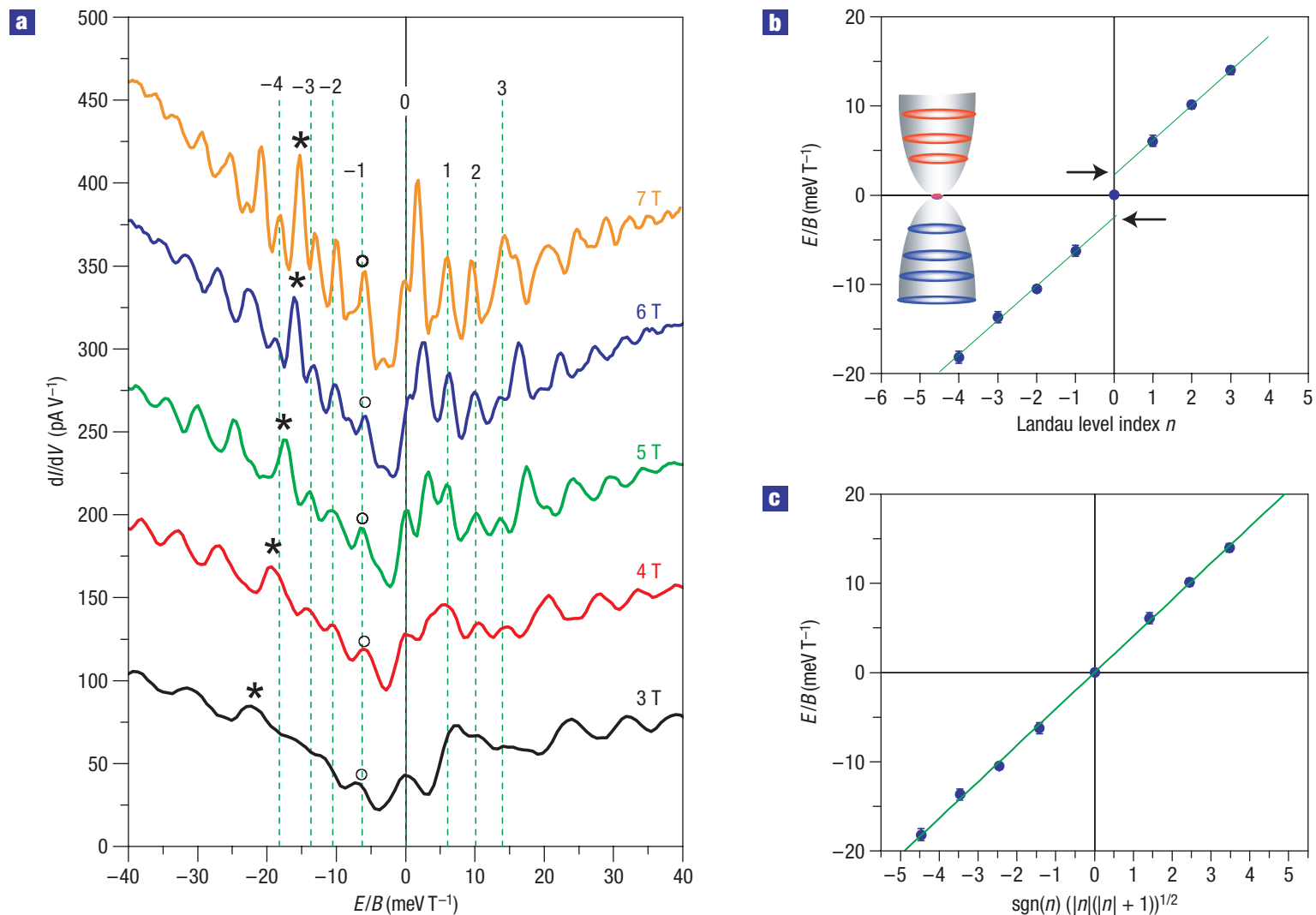

Figure 3 Landau levels of massive DFs. a, Tunnelling spectra plotted against the reduced energy $E / B$. The energy origin was shifted to peak $A$ in Fig. 1 . The peaks marked with circles are aligned after scaling, whereas those marked with stars are not. All aligned peaks are labelled with sequential LL indices. The spectra are shifted vertically by $70 \mathrm{pAV}^{-1}$ for clarity. b, Scaled energy plotted against $\mathrm{LL}$ index. Solid lines represent the $\mathrm{LL}$ spectrum expected for normal massive fermions. The arrows show two missing $n=0$ levels. Inset: Landau-level energy sequence of massive DFs superposed on the zero-field dispersion. c, The scaled energy is shown to be linear in $\operatorname{sgn}(n)(|n|(|n|+1))^{1 / 2}$, as expected for massive chiral fermions. The solid line is a linear fit with effective mass $m^{*}=0.028 m_{\mathrm{e}}$.
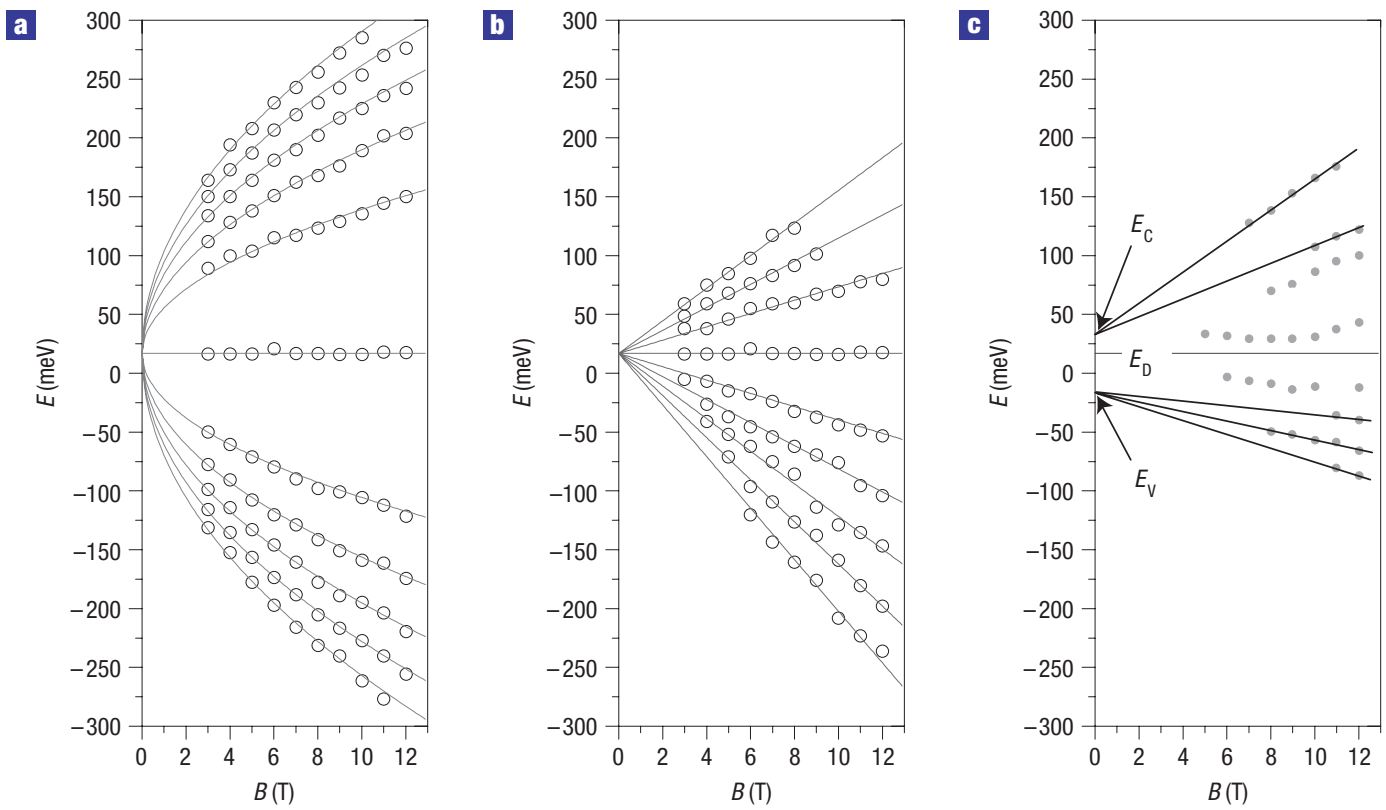

Figure 4 Classification of LLs in magnetic field up 12 T. a, Massless DFs. Solid lines represent fits of the spectra in Fig. 2 with equation (2). b, Massive DFs. Solid lines represent fits of the spectra in Fig. 3 with equation (3). c, Unidentified levels. The solid lines show that the LLs do not coalesce into a single energy at zero field. 
in $m^{*}$ of the massive DFs (see Supplementary Information, Fig. S3). This finding demonstrates that the observed twodimensional behaviour at large sample-tip distance is not a simple charging effect. Indeed, if this were the case, the two-dimensional quasiparticles would be observable in the scanning tunnelling spectra of all types of graphite, but their absence from Kish graphite $^{18}$ suggests that material structure plays an important role. Thus it was recently proposed that in the presence of stacking faults, or when the stacking is rhombohedral rather than Bernal, the surface layers of graphite become well decoupled from the bulk, giving rise to both massless and massive quasiparticles (F. Guinea, private communications). This is consistent with our observations. However, more detailed theoretical modelling is needed to understand the scanning tunnelling spectra and the twodimensional nature of the quasiparticles on the surface of graphite.

\section{METHODS}

Experiments were carried out on a home-built low-temperature high-magnetic-field scanning tunnelling microscope recently developed in our group. The controller was a commercial SPM 1000 controller from RHK Technologies. The samples were HOPG cleaved in air, and the scanning tunnelling microscope tips were obtained from mechanically cut Pt-Ir wire. The tip-sample distance was set by a tunnelling current of $25 \mathrm{pA}$ with sample bias voltage of $300 \mathrm{mV}$ unless otherwise specified. We use the small set current to minimize the effect of the tip on the tunnelling spectra. The data shown here represent the average of 256 spectra measured over an area of $50 \times 50 \mathrm{~nm}^{2}$. The $\mathrm{d} I / \mathrm{d} V$ measurements were carried out with a lock-in technique using a $450 \mathrm{~Hz}$ a.c. modulation of the bias voltage with an amplitude of $5 \mathrm{mV}$. The magnetic field was applied perpendicular to the sample surface with a superconducting magnet working in persistent mode.

Received 1 December 2006; accepted 29 May 2007; published 8 July 2007.

\section{References}

1. Novoselov, K. S. et al. Electric field effect in atomically thin carbon films. Science 306, 666-669 (2004).

2. Novoselov, K. S. et al. Two dimensional atomic crystals. Proc. Natl Acad. Sci. USA 102 10451-10453 (2005)

3. Zheng, Y. \& Ando, T. Hall conductivity of a two-dimensional graphite system. Phys. Rev. B 65, 245420 (2002).

4. Novoselov, K. S. et al. Two-dimensional gas of massless Dirac fermions in graphene. Nature $\mathbf{4 3 8}$ 197-200 (2005).

5. Zhang, Y., Tan, Y. W., Stormer, H. L. \& Kim, P. Experimental observation of the quantum Hall effect and Berry's phase in graphene. Nature 438, 201-204 (2005).
6. Gusynin, V. P. \& Sharapov, S. G. Unconventional integer quantum Hall effect in graphene. Phys. Rev. Lett. 95, 146801 (2005).

7. Castro Neto, A. H., Guinea, F. \& Peres, N. M. R. Edge and surface states in the quantum Hall effect in graphene. Phys. Rev. B 73, 205408 (2006).

8. Novoselov, K. S. et al. Unconventional quantum Hall effect and Berry's phase of $2 \pi$ in bilayer graphene. Nature Phys. 2, 177-180 (2006).

9. Nilsson, J., Castro Neto, A. H., Peres, N. M. R. \& Guinea, F. Electron-electron interactions and the phase diagram of a graphene bilayer. Phys. Rev. B 73, 214418 (2006).

10. MaCann, E., Fal'ko, V. I. \& Zhang, Y. Landau-level degeneracy and quantum Hall effect in a graphite bilayer. Phys. Rev. Lett. 96, 086805 (2006).

11. Peres, N. M. R., Guinea, F. \& Castro Neto, A. H. Electronic properties of disordered two-dimensional carbon. Phys. Rev. B 73, 125411 (2006).

12. Semenoff, G. W. Condensed matter simulation of a three-dimensional anomaly. Phys. Rev. Lett. 53, 2449-2452 (1984).

13. Haldane, F. D. M. Model for a quantum Hall effect without Landau levels: condensed matter realization of the parity anomaly. Phys. Rev. Lett. 61, 2015-2018 (1988).

14. Kopelevich, Y. et al. Reentrant metallic behavior of graphite in the quantum limit. Phys. Rev. Lett. 90, 156402 (2003).

15. Luk'yanchuk, I. A. \& Kopelevich, Y. Dirac and normal fermions in graphite and graphene: implications of the quantum Hall effect. Phys. Rev. Lett. 97, 256801 (2006).

16. Ishigami, M., Chen, J. H., Cullen, W. G., Fuhrer, M. S. \& Williams, E. D. Atomic structure of graphene on $\mathrm{SiO}_{2}$ Nano Lett. (in the press) (2007).

17. Martin, J. et al. Observation of electron-hole puddles in graphene using a scanning single electron transistor. Preprint at <http://arxiv.org/abs/0705.2180> (2007).

18. Matsui, T. et al. STS observations of Landau levels at graphite surfaces. Phys. Rev. Lett. 94 226403 (2005).

19. Niimi, Y., Kambara, H., Matsui, T., Yoshioka, D. \& Fukuyama, H. Real-space imaging of alternate localization and extention of quasi-two-dimensional electronic states at graphite surfaces in magnetic fields. Phys. Rev. Lett. 97, 236804 (2006).

20. Zhou, S. Y. et al. A. First direct observation of Dirac fermions in graphite. Nature Phys. 2, 595-599 (2006)

21. Dresselhaus, G. Graphite Landau levels in the presence of trigonal warping. Phys. Rev. B 10, 3602-3609 (1974).

22. Galt, J. K., Yager, W. A. \& Dail, H. W. Jr.. Cyclotron resonance effects in graphite. Phys. Rev. 103, 1586-1587 (1956).

23. Soule, D. E. Magnetic field dependence of the Hall effect and magnetoresistance in graphite single crystals. Phys. Rev. 112, 698-707 (1958).

24. Zhang, Y. B., Small, J. P., Amori, M. E. S. \& Kim, P. Electric field modulation of galvanomagnetic properties of mesoscopic graphite. Phys. Rev. Lett. 94, 176803 (2005)

25. Guinea, F., Castro Neto, A. H. \& Peres, N. M. R. Electronic states and Landau levels in graphene stacks. Phys. Rev. B 73, 245426 (2006).

26. Wallace, P. R. The band theory of graphite. Phys. Rev. 71, 622-634 (1947).

27. Morozov, S. V. et al. Two-dimensional electron and hole gases at the surface of graphite. Phys. Rev. B 72, 201401 (2005).

\section{Acknowledgements}

We acknowledge discussions with A. V. Balatsky, A. H. Castro Neto, P. Coleman, J. C. Davis, X. Du, A. Ermakov, A. Geim, F. Guinea, E. Garfunkel, P. Kim and I. Skachko. Work supported by NSF-DMR-0456473 and by DOE DE-FG02-99ER45742.

Correspondence and requests for materials should be addressed to E.Y.A.

Supplementary Information accompanies this paper on www.nature.com/naturephysics.

Competing financial interests

The authors declare no competing financial interests.

Reprints and permission information is available online at http://npg.nature.com/reprintsandpermissions/ 